\title{
Comparação de algoritmos genéticos na solução de problemas de aporte de cargas de poluição em rios
}

\author{
Juliane A. Ferreiralt \\ DI/UFES, Vitória, ES \\ Selena L. Carvalhdp \\ LABHIDRO/UFES, Vitória, ES \\ Maria Cristina Range $]^{3}$ \\ DI/UFES, Vitória, ES \\ Lucia Catabriga" \\ DI/UFES, Vitória, ES
}

Resumo. Os rios brasileiros são classificados em classes de qualidade de água, de acordo com o Enquadramento de Corpos D'água. Nos últimos anos, algoritmos evolutivos vem sendo utilizados para encontrar a eficiência mínima de redução de poluentes garantindo o enquadramento de rios na legislação vigente. Este trabalho tem por objetivo comparar três algoritmos na busca de soluções ótimas: algoritmo genético considerando mutação, algoritmo genético considerando mutação e elitismo e algoritmo genético das chaves aleatórias viciadas (BRKGA). Os algoritmos são comparados no estudo de qualidade de água na bacia hidrográfica do rio Pardo, localizada no estado do Espírito Santo.

Palavras-chave. Poluição Em Rios, Algoritmos Genéticos, Mutação, Elitismo, BRKGA.

\section{Introdução}

Os padrões brasileiros de qualidade de água foram fixados pela Resolução do Conselho Nacional de Meio Ambiente (Conama) $\mathrm{n}^{0} 357$ de 2005, a qual foi posteriormente complementada pela resolução Conama $\mathrm{n}^{\mathrm{O}} 430$ de 2011, estabelecendo limites de concentração para vários parâmetros de qualidade de água. Todavia, a qualidade de água nos rios brasileiros frequentemente está em desacordo com a legislação vigente, violando o referido padrão de qualidade, e essa violação ocorre principalmente devido a aportes de cargas poluentes aos cursos d'água.

A poluição em recursos hídricos ou poluição hídrica, pode ser classificada quanto a origem das fontes das cargas poluidoras, sendo dita pontual quando o lançamento de carga ocorre de modo concentrado e em localidade específica, como por exemplo, o esgoto de uma cidade. E quando essa carga poluente não possui uma fonte ou localidade específica, como os poluentes que se encontram presentes nas enxurradas, a poluição é dita difusa [6].

Existem inúmeros métodos e tecnologias de tratamento para a redução e controle da poluição hídrica, entretanto, o ponto inicial para otimização destes sistemas consiste em identificar os valores mínimos de redução necessários para o atendimento dos limites de concentração (de cada poluente) vinculados ao padrão de qualidade de água, de modo que não ocorra violação da legislação

\footnotetext{
1 juliane.ferreira@edu.ufes.br.

2 selenaesa@gmail.com.

3 crangel@inf.ufes.br.

${ }^{4}$ luciac@inf.ufes.br.
} 
ambiental vigente. Para a identificação destes percentuais de redução, comumente são empregados métodos de busca, especialmente meta-heurísticas, com o objetivo de obter soluções próximas às soluções ótimas [4].

Dentre as meta-heurísticas comumente empregadas para o problema acima apresentado, os algoritmos genéticos destacam-se, tendo como característica principal a simulação da evolução das espécies como método de busca pelo indivíduo ou indivíduos ótimos. Sendo, portanto, um método populacional estocástico de busca direta com operadores probabilísticos 2,4 .

O presente trabalho considera a implementação de um conjunto de modelos ambientais para redução de cargas poluidoras em bacias hidrográficas comparando três algoritmos genéticos na busca de soluções ótimas: algoritmo genético com mutação, denominado de AG1 [5], algoritmo genético com mutação e elitismo, denominado AG2 e algoritmo genético das chaves aleatórias viciadas ou Biased Random-Key Genetic Algorithm (BRKGA) [3]. Os algoritmos são comparados, através da qualidade das soluções encontradas e o tempo computacional necessário, no estudo de qualidade de água na bacia hidrográfica do rio Pardo, localizada no estado do Espírito Santo.

\section{Aplicação física}

A bacia hidrográfica do rio Pardo, afluente do rio Itapemirim, está localizada quase totalmente no estado do Espírito Santo. Uma sucinta caracterizaçao da bacia pode ser vista na Figura 1a. Os esgotos domésticos dos núcleos urbanos dos municípios de Ibatiba e Iúna são caracterizadas

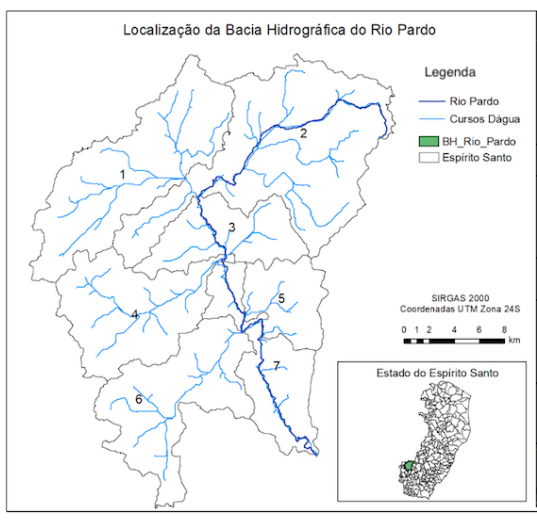

(a) Localização da bacia.

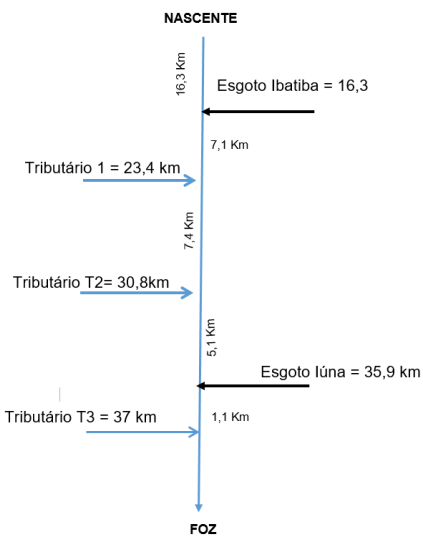

(b) Diagrama unifilar.

Figura 1: Bacia do Rio Pardo.

como fontes pontuais, como mostra o diagrama unifilar da Figura 1b. Para a caracterização de fontes difusas a bacia hidrográfica é subdivida em sete unidades, denominadas de sub-bacias e identificadas pelos algarismos de 1 a 7 na Figura 1a. Para cada uma destas fontes são levantados e/ou estimadas a produção de cargas poluentes 1 .

Para simular o comportamento dos poluentes no curso d'água, utilizamos o modelo matemático QUAL-UFMG 8], sendo simulados os parâmetros: Demanda Biológica de Oxigênio (DBO5), Nitrogênio Amoniacal (NH3), Nitrito (NO), Oxigênio Dissolvido (OD) e Fósforo (P). O modelo é composto por um sistema de equações diferenciais de primeira ordem do tipo:

$$
\frac{d Y}{d t}=F(Y, t)
$$


onde o vetor incógnita é definido por $Y=(D B O 5, N H 3, N O, O D, P)^{t}$ e vetor $F(Y, t)$, com componentes $f_{i}=(Y, t)$, é definido por funções lineares que relacionam as concentrações $(\mathrm{em} m g / L$ ) dos parâmetros físicos de interesse. Todos os detalhes da formulação matemática do modelo podem ser consultados em [1. O sistema (1) é aproximado pelo método de Euler. Para tal, é considerada uma divisão do domínio em um conjunto de células e aproximações em série de Taylor até a primeira ordem para obter os valores aproximados dos parâmetros de interesse em cada célula 8 . A resolução CONAMA $n^{0} 357$ estabelece valores de referência $(\mathrm{em} \mathrm{mg} / \mathrm{L}$ ) na concentração desses parâmetros, sendo que as concentrações referente a Classe $2^{5}$ são dadas por:

$$
D B O 5 \leq 5 ; O D \geq 5 ; N O \leq 1 ; P \leq 0,1 \text { e } N H 3 \leq 3,7 \text { para } \mathrm{pH} \leq 7 .
$$

As cargas difusas são principalmente substâncias que são lixiviadas pela enxurrada (escoamento superficial) durante eventos de chuva. Assim, foi necessário implementar modelos de estimativa de volume de escoamento superficial e de cargas difusas para simular a produção destas cargas, sendo então utilizados os métodos Soil Conservation Service do Natural Resources Conservation Service (NRCS/EU) e o método da Concentração Média de Evento (CME) 7]. Ressaltamos, também, que todos os detalhes da formulação numérica podem ser consultados em 1$]$.

\section{Algoritmos Genéticos}

Nesta seção apresentamos os três algoritmos genéticos estudados neste trabalho: AG1, AG2 e BRKGA. Cada um desses algoritmos possui duas versões: o Algoritmo Genético de Otimização de Fontes Pontuais (AGOFP) e o Algoritmo Genético de Otimização de Fontes Difusas (AGOFD). Um AG é composto por uma população de cromossomos (soluções), os quais são submetidos a uma função de avaliação, função objetivo (FO), considerando um conjunto de restrições a serem atendidas [5].

O AGOFP busca identificar a eficiência mínima de remoção que uma determinada Estação de Tratamento de Esgoto (ETE) deve ter para não violar os limites de concentração de classe no curso d'água que recebe o efluente tratado. A melhor solução é aquela que apresenta a menor eficiência de remoção atendendo aos padrões de classe de qualidade de água. Portanto, na versão da poluição pontual, o cromossomo é representado por um vetor composto por $n_{p}$ números randômicos gerados no intervalo $\left[E_{m} ; 0,9\right]$, sendo $E_{m}$ a eficiência mínima ${ }^{6}$ e $n_{p}$ o número de lançamentos de esgoto na bacia hidrográfica. Para o rio Pardo, consideramos duas entradas pontuais de fontes poluidoras - observe a Figura 1b. As restrições são os limites de qualidade de água fixados pela resolução Conama 357/2005, representados na equação (2). Para este algoritmo, o parâmetro otimizado foi a DBO5, uma vez que a legislação ambiental estabelece a obrigatoriedade de tratar esse parâmetro.

Por sua vez, o AGOFD identifica o percentual mínimo a ser removido nos parâmetros DBO5, $\mathrm{NH} 3$, NO e P, considerando como restrição os mesmos limites de classe. Consequentemente, o cromossomo é definido como uma matriz $n_{d} \times 4$ de valores randômicos no intervalo $[0 ; 0,9]$, onde $n_{d}$ representa o número de entradas de fonte difusas de poluição. Para o rio Pardo, $n_{d}$ representa o número de sub-bacias, conforme Figura 1a.

Nas duas versões do algoritmo, a população inicial é formada a partir da geração aleatória de soluções até que $n_{\text {sol }}$ soluções válidas sejam geradas. Neste estudo, consideramos que quanto menor a eficiência da tecnologia de tratamento, menor será o seu custo de implementação. Assim,

\footnotetext{
${ }^{5}$ A Resolução Conama 357/2005 estabelece as seguintes classes de qualidade: Especial, 1, 2, 3 e 4. Para cada classe são estabelecidos os possíveis usos atribuídos a estas águas, e em função destes usos são estabelecidos os limites de concentração dos parâmetros de qualidade de água.

${ }^{6}$ Referente aos limites mínimos de eficiência que as ETEs devem possuir, os quais foram fixados pela CONAMA $430 / 2011$.
} 
minimizar a eficência representa a solução desejada. As funções objetivo de AGOFP e AGOFD são representadas pelas equações (3) e (4), respectivamente.

$$
\begin{aligned}
\min _{j=1, n_{\text {sol }}} F_{P}(j)= & \sum_{i=1}^{n_{p}} E^{j}(i) \\
\min _{j=1, n_{\text {sol }}} F_{D}(j)= & \sum_{i=1}^{n_{d}}\left[\left(W_{D B O_{5}} R_{D B O_{5}}^{j}(i)\right)+\left(W_{N H_{3}} R_{N H_{3}}^{j}(i)\right)+\right. \\
& \left.\left(W_{N O} R_{N O}^{j}(i)\right)+\left(W_{P} R_{P}^{j}(i)\right)\right]
\end{aligned}
$$

onde $E^{j}(i)$ é a $j$-ésima eficiência de tratamento da $i$-ésima fonte poluidora pontual relativa a DBO5; $R_{*}^{j}$ é a $j$-ésima remoção das cargas poluentes na $i$-ésima sub-bacia, $W_{*}$ é o peso do parâmetro $R_{*}^{j}$, respectivamente, para DBO5, NH3, NO e P.

A partir da população inicial, as novas populações são geradas por meio de operadores genéticos. O cruzamento é a geração de um novo indivíduo (filho) que carrega material genético de outros dois (os pais). Nos três algoritmos, a mutação é caracterizada pela substituição de indivíduos de baixa qualidade por indivíduos gerados randomicamente.

No algoritmo BRKGA o cromossomo é definido como um conjunto de chaves aleatórias, ou seja, números randômicos gerados no intervalo $[0,1]$. Ao ser criado, o indivíduo é então decodificado para dentro do espaço de busca em questão: $\left[E_{m}, 0,9\right]$ e $[0 ; 0,9]$ referentes, respectivamente, as poluições pontual e difusa. O decodificador faz uso de regra de três simples, encontrando o valor do gene correspondente à chave. No BRKGA é utilizada a técnica denominada elitismo - a hierarquização das soluções de acordo com os valores obtidos pela aplicação da FO. As soluções que apresentam menores valores de eficiência de remoção constituem um conjunto denominado elite. Portanto, a nova população passa a ser formada pelo conjunto elite, pelos indivíduos resultantes dos cruzamentos e pelas soluções oriundas da etapa de mutação. No cruzamento, a escolha dos dois pais é aleatória, sendo um escolhido dentre o conjunto elite e o outro escolhido considerando toda a população. Em seguida, todos os cromossomos da nova população são traduzidos pelo decodificador para novo processo de cruzamento e mutação. Este processo é repetido um número fixo de iterações $\left(n_{\text {iter }}\right)$. A melhor solução dentre todas as iterações é a solução apresentada pelo algoritmo.

No algoritmo AG1 a nova população é composta apenas pelos mutantes e pelos filhos resultantes da etapa de cruzamento, sem considerar elitismo. Por sua vez, o algoritmo AG2 é similar ao BRKGA sem considerar o decodificador, ou seja, os individuos são gerados diretamente dentro do espaço de busca dos problemas. Os algoritmos AG1, AG2 e BRKGA nas suas duas versões (AGOFP e AGOFD) foram implementados na linguagem Python e compõe o Sistema Integrado de Modelagem de Poluição Pontual e Difusa (SIMPPOD) $]^{7}$ em desenvolvimento pelas autoras do trabalho.

\section{Experimentos Numéricos}

Nesta seção apresentamos os experimentos realizados na Bacia do rio Pardo utilizando valores reais de uso e ocupação referentes ao ano de 2015, disponibilizados pelo GEOBASES 8 Após um conjunto preliminar de testes, em todos os nossos experimentos são considerados: um número fixo de iterações $\left(n_{\text {iter }}=100\right)$, sendo cada população de tamanho fixo $n_{\text {sol }}=50$ indivíduos; os pesos $W_{*}$

\footnotetext{
${ }^{7} \mathrm{O}$ vídeo do Youtube apresentando a interface do programa SIMPPOD pode ser consultado em https://www. youtube. com/watch?v=Tn9S11QIA6U

${ }^{\circledR}$ Sistema Integrado de Bases Geoespaciais do Estado do Espírito Santo (https://geobases.es.gov.br)
} 
da Equação (4) iguais a 0,25; a porcentagem do conjunto elite de $30 \%$ e do conjunto de mutantes de $10 \%$. Os valores adotados para estas simulações foram baseados nos resultados encontrados no trabalho de 3 Com o objetivo de minimizar os efeitos aleatórios dos algoritmos, realizamos um total de 10 repetições dos testes para todos os métodos considerados e todos os resultados a seguir mostram as médias obtidas nas 10 execuções.

A Tabela 1 apresenta a média e o desvio padrão do número de inválidos da população inicial, dos cruzamentos e dos mutantes para os algoritmos AG1, AG2 e BRKGA nas versões AGOFP e AGOFD. Claramente os números de inválidos para as versões relativas à poluição difusa são mais elevados. Essa característica é esperada uma vez que a poluição difusa contém um número maior de poluentes, um número maior de fontes de poluição (sete sub-bacias) e também pela magnitude das entradas correspondentes a este tipo de poluição. Os números de inválidos gerados nos cruzamentos para as versões pontuais são sempre nulos, provavelmente devido a simplicidade da aplicação que contém apenas duas fontes pontuais. O desvio padrão do número de inválidos para a população inicial é mais elevado quando comparado aos demais. Enquanto que os desvios do número dos inválidos dos cruzamentos e dos mutantes corroboram a regularidade dos algoritmos genéticos na geração de novas soluções.

Tabela 1: Média e Desvio Padrão dos conjuntos de inválidos gerados na criação da população inicial, nos cruzamentos e na criação do grupo de mutantes no AG1, AG2 e BRKGA, para AGOFP e AGOFD.

\begin{tabular}{cc|cc|cc}
\hline Método & Conjunto & AGOFP & & AGOFD & \\
& & Média & DP (\%) & Média & DP (\%) \\
\hline \multirow{3}{*}{ AG1 } & População Inicial & 7569,75 & 8,41 & 250918,00 & 9,11 \\
& Cruzamentos & 0,00 & 0,00 & 4919,63 & 0,94 \\
& Mutantes & 76081,63 & 3,48 & 2631513,38 & 3,22 \\
\hline \multirow{2}{*}{ AG2 } & População Inicial & 7779,75 & 11,15 & 272940,38 & 11,63 \\
& Cruzamentos & 0,00 & 0,00 & 3394,38 & 1,78 \\
& Mutantes & 76069,00 & 2,03 & 2671779,00 & 2,77 \\
\hline \multirow{3}{*}{ BRKGA } & População Inicial & 7779,25 & 9,61 & 291589,25 & 7,25 \\
& Cruzamentos & 0,00 & 0,00 & 3364,13 & 1,02 \\
& Mutantes & 75252,13 & 3,31 & 2640485,88 & 3,93 \\
\hline
\end{tabular}

A média e o desvio padrão das funções objetivos para os três métodos aplicados às poluições pontual e difusa são apresentados na Tabela 2 Para a poluição pontual, podemos notar que as médias das FOs não variam para os três algoritmos. Entretanto, para a poluição difusa as médias das FOs para os algoritmos AG2 e BRKGA foram praticamente a metade daquela obtida para o algoritmo AG1. O desvio padrão das FOs para os algoritmos AGOFP foram expressivamente menores do que para os algoritmos AGOFD.

Tabela 2: Média e Desvio Padrão da FO no AG1, AG2 e BRKGA, para AGOFP e AGOFD.

\begin{tabular}{c|cc|cc}
\hline Método & AGOFP & & AGOFD & \\
& Média & DP (\%) & Média & DP (\%) \\
\hline AG1 & 1,62 & 0,35 & 12,47 & 3,18 \\
AG2 & 1,62 & 0,02 & 6,29 & 1,60 \\
BRKGA & 1,62 & 0,01 & 6,44 & 4,34 \\
\hline
\end{tabular}

A Tabela 3 apresenta as eficiências de DBO5 e OD para a poluição pontual e eficiências de DBO5, NH3, NO e P para a difusa. Os três algoritmos geram eficiências praticamente iguais com 
desvios padrão muito pequenos para os dois parâmetros de qualidade de água avaliados para a poluição pontual. O mesmo não ocorre para a poluição difusa, pois os resultados do AG1 são inferiores aos resultados do AG2 e BRKGA, que apresentam soluções semelhantes. Isso indica que a estratégia do elitismo utilizada no cruzamento, presente em ambos, pode gerar bons resultados para problemas de otimização de cargas poluentes em rios. A similaridade nos resultados dos AG2 e

Tabela 3: Média e Desvio Padrão das eficiências de DBO5 e OD; e da soma das eficiências de DBO5, NH3, NO e P no AG1, AG2 e BRKGA, para AGOFP e AGOFD.

\begin{tabular}{c|ccc|ccc}
\hline Método & AGOFP & & \multicolumn{3}{c}{ AGOFD } \\
& Parâmetro & Média & DP (\%) & Parâmetro & Média & DP (\%) \\
\hline \multirow{4}{*}{ AG1 } & DBO5 & 0,90 & 0,02 & DBO5 & 5,08 & 4,23 \\
& & & & NH3 & 2,41 & 17,25 \\
& OD & 0,73 & 0,80 & NO & 2,57 & 18,44 \\
& & & & P & 2,48 & 21,77 \\
\hline \multirow{4}{*}{ AG2 } & DBO5 & 0,90 & 0,00 & DBO5 & 4,50 & 2,45 \\
& & & & NH3 & 0,68 & 12,68 \\
& OD & 0,72 & 0,04 & NO & 0,73 & 14,87 \\
& & & & P & 0,36 & 25,39 \\
\hline \multirow{3}{*}{ BRKGA } & DBO5 & 0,90 & 0,00 & DBO5 & 4,44 & 2,41 \\
& \multirow{2}{*}{ OD } & \multirow{2}{*}{0,72} & 0,03 & NH3 & 0,70 & 16,46 \\
& & & & NO & 0,71 & 14,05 \\
& & & & P & 0,43 & 20,14 \\
\hline
\end{tabular}

BRKGA sugere que a estratégia de chaves aleatórias, presente no BRKGA, não auxilia na melhoria dos resultados deste algoritmo. De modo geral, o AG2 foi mais eficiente na minimização da função objetivo, apresentando portanto, solução melhor que a solução encontrada pelo BRKGA. E quanto aos desvios padrão, estes são expressivamente maiores do que aqueles obtidos para a poluição pontual, exceto para a DBO5. Este fato ocorre devido a complexidade nos processos ambientais referentes aos parâmetros NH3 e NO, e em função das características randômicas presentes nestes algoritmos. Entretanto, quando se trata dos desvios padrão das FOs (Tabela 22 observa-se que estes mostram que, de modo geral, as soluções obtidas nos testes tem características similares quando se trata do objeto de otimização, ou seja, o conjunto de eficiências de todos os parâmetros.
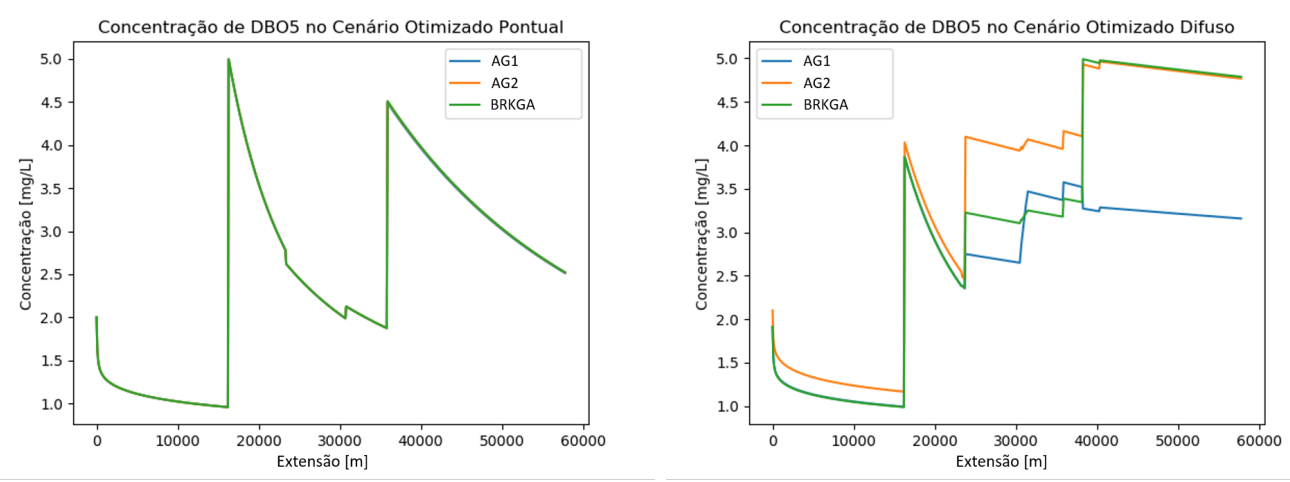

Figura 2: Comportamento das eficiências de DBO5 para as poluições pontual (à esquerda) e difusa (à direita) pelos algoritmos AG1, AG2 e BRKGA. 
Os comportamentos das eficiências de DBO5 ao longo da extensão do rio Pardo para as poluições pontual e difusa podem ser observadas na Figura 2. Para a poluição pontual não há variação no comportamento para os três algorimos (gráfico à esquerda), já para a poluição difusa as eficiências de DBO5 apresentam comportamentos diferentes (gráfico à direita). O perfil resultante do AG2 apresentou maiores concentrações de DBO5 entre os quilômetros 23 e 39 (aproximadamente), indicando que para estes trechos este algoritmo encontrou menores eficiências de remoção. Todavia, o menor somatório de eficiências referente ao parâmetro DBO5 foi encontrado pelo BRKGA, como pode ser observado na Tabela 3

\section{Conclusões}

Este trabalho apresenta uma aplicação de algoritmos genéticos para encontrar a eficiência mínima de redução de cargas difusas e pontuais de poluição em rios, garantindo o atendimento da legislação vigente. O artigo apresenta um estudo comparativo de algoritmos genéticos com mutação (AG1), mutação e elitismo (AG2) e com chaves aleatórias viciadas (BRKGA). Os experimentos apresentam o estudo de qualidade de água na bacia hidrográfica do rio Pardo, localizada no estado do Espírito Santo. Para o conjunto de testes realizados concluímos que para a poluição pontual os três algoritmos encontraram soluções similares dado a simplicidade deste sistema, enquanto que para a poluição difusa, que gera um sistema mais complexo, os algoritmos BRKGA e AG2 encontraram soluções superiores e bastante próximas. De modo geral, o AG2 foi mais eficiente na minimização do conjunto de eficiências, apresentando portanto, soluções ligeiramente melhores que as soluções encontradas pelo BRKGA.

\section{Referências}

[1] Carvalho, S. L. Sistema De Suporte A Decisão Para Planejamento, Controle E Redução De Cargas Difusas E Pontuais Em Bacias Hidrográficas, Tese de Doutorado, UFES, 2019.

[2] Goldberg, D. E. Genetic algorithms in search, optimization, and machine learning, 1a. edição. Addison-Wesley, Boston, 1989.

[3] Gonçalves, J. F. e Resende, M. G. C. Biased random-key genetic algorithms for combinatorial optimization, Journal of Heuristics, 17:487-525, 2011. DOI:10.1007/s10732-010-9143-1.

[4] Hernandez, E. A. e Uddameri, V. An Assessment Of Optimal Waste Load Allocation And Assimilation Characteristics In The Arroyo Colorado River Watershed, TX Along The US-Mexico Border, Clean Technologies and Environmental Policy, 15:617-630, 2013. DOI:10.1007/s10098012-0546-6.

[5] Linden, R. Algoritmos Genéticos, 3a. edição. Ciência Moderna, Rio de Janeiro, 2012.

[6] Novotny, V. Water quality: diffuse pollution and watershed management, 2a. edição. John Wiley, New Jersey, 2003.

[7] Tucci, C. E. M. Hidrologia: ciência e aplicação, 3a. edição. Editora da UFRGS: ABRH, Porto Alegre, 2004.

[8] Von Sperling, M. Introdução À Qualidade Das Águas E Ao Tratamento De Esgotos, 4a. edição. Departamento de Engenharia Sanitária e Ambiental, UFMG, Belo Horizonte, 2014. 